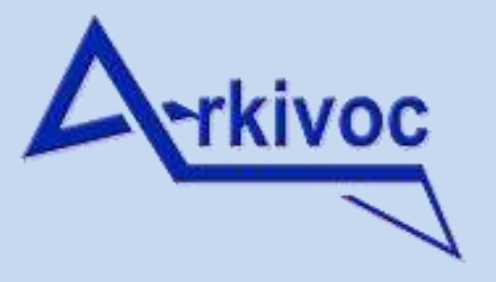

Archive for

Organic Chemistry
The Free Internet Journal

for Organic Chemistry
Paper

Arkivoc 2020, part iv, 59-66

\title{
Computational study of phenyliodine diacetate intermediates during Lewis acid activation with TMSOTf
}

Taro Jones, Jennifer Noorollah, Nirvanie Singh, Nicholas R. Spatola, Andrew Zhang, Azka Chaudhry, and I. F. Dempsey Hyatt

Adelphi University, Department of Chemistry and Biochemistry, 1 South Ave., Garden City, NY 11530 Email: ihyatt@adelphi.edu

Received 12-04-2020

Accepted 02-02-2021

Published on line $\quad 02-08-2021$

\section{Abstract}

A method of increasing the reactivity of phenyliodine(III) diacetate (PIDA) involves the addition of a Lewis acid. The acid-activation of PIDA was investigated toward the goal of elucidating relevant intermediates that participate in other mechanisms such as the transmetallation of hypervalent iodine compounds with metalloids and diaryliodane synthesis. Our computational analysis focused on the reaction coordinate of PIDA reacting with TMSOTf. Acetate-triflate substitutions were found to be thermodynamically preferred over PIDA, and the possibility of a four-coordinate iodine intermediate is evaluated. Other structural and electronic considerations involving trans-influence, $\sigma$-hole, and the 3-center-4-electron (3c-4e) bond are presented.

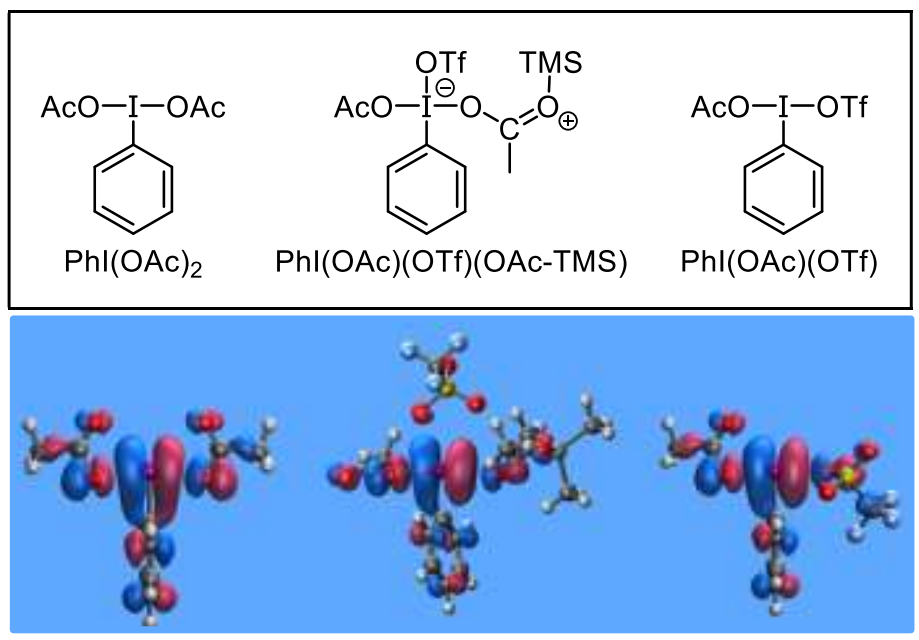

Keywords: Hypervalent iodine, 3c-4e bond, sigma-hole, PIDA, trans-influence 


\section{Introduction}

\section{Computational study of phenyliodine diacetate intermediates during Lewis acid activation with TMSOTf}

Hypervalent iodine (HVI) has continued to garner the interest within the organic chemistry community. ${ }^{[1-5]}$ The ubiquity of hypervalent iodine reagents in chemical reactions is related to iodine's accessible oxidations states, mainly iodine(III) and iodine(V). Typically, iodine(III) reagents rely on a Lewis-acid activation, and iodine(V) reagents are used in oxidations. ${ }^{[6-8]}$ Due to properties such as the trans-influence, $\sigma$-hole, and the 3-center-4electron ( $3 \mathrm{c}-4 \mathrm{e}$ ) bond, devising definitive reaction mechanisms based on HVI-(III) intermediates can be challenging. ${ }^{[9,10]}$ The lack of knowledge of iodine(III) is only magnified when analyzed from a computational standpoint as many structural conformers are possible along reaction coordinates. ${ }^{[11,12]}$ The research presented herein analyzes the intermediates in the reaction between phenyliodine(III) diacetate (PIDA) and trimethylsilyl triflate (TMSOTf) toward the goal of contributing potential HVI-(III) structures that participate in a multitude of reactions across organic synthesis. ${ }^{[13-15]}$

PIDA can be considered a staple among HVI reagents, be it either as a direct reactant or as an intermediate in synthesizing the intended $\lambda^{3}$-iodoarene. ${ }^{[8,16]}$ Using PIDA derivatives also aligns with a more sustainable route, as a vast majority of $\mathrm{HVI}$ reactions result in the reduction of the iodoarene as a byproduct. ${ }^{[6]}$ Oxidation and reoxidation of the ${ }^{(1)}$ byproducts can be accomplished separately from the main synthesis, ${ }^{[16]}$ or as a parallel process. ${ }^{[17]}$ Lewis acid activation of PIDA is usually performed by the addition of $\mathrm{BF}_{3} \cdot \mathrm{Et}_{2} \mathrm{O}$ or $\mathrm{TMSOTf}$ in a halogenated solvent. ${ }^{[16]}$ The mechanism likely next involves the Lewis acid coordination to the acetate, but from there, the central question is whether $\mathrm{Phl}(\mathrm{OTf})_{2}$ or $\mathrm{Phl}(\mathrm{OAc})(\mathrm{OTf})$ is the activated species.

The reactivity of Weiss' reagent, $\left[\mathrm{PhI}(\mathrm{pyr})_{2}\right]^{2+}$, a compound of recent interest synthesized by PIDA-activation with TMSOTf, ${ }^{[18,19]}$ led the Hyatt group to the state-of-the-art mechanistic investigation by Dutton et al. ${ }^{[7]}$ Dutton et al. originally proposed $\mathrm{Phl}(\mathrm{OTf})_{2}$ was the active species involved with the generation of $\mathrm{Phl}(\mathrm{py})_{2}(\mathrm{OTf})_{2}$ and provided our study with an excellent starting point to extend the theoretical work to other participating intermediates during activation. Recently, Dutton et al. built on their past work by publishing an article titled, Phl(OTf) 2 Does Not Exist (Yet). ${ }^{[20,21]}$ In the article, the Dutton group presents spectroscopic data supporting their title's claim and thus seeming to contradict their original 2012 article; but in later work the Dutton group noted several spectroscopic inconsistencies concerning the existence of Phl(OTf $)_{2 .}{ }^{[22,23]}$ To support Dutton's claim, we present energies and molecular orbital data to show the active species is Phl(OAc)(OTf).

The majority of the calculations were performed using an effective core potential for iodine, while other elements were treated with the $6-311++g(d, p)$ basis set. In a pivotal study by Mocci and Cerioni et al., the solution structures of $\lambda^{3}$-iodanes by ${ }^{17} \mathrm{O}$ NMR and DFT were performed in which the authors rationalized the in vacuo geometry optimizations by citing the low dielectric constant of chloroform as a justification for negligible differences. Our study relied on the same justification and were also optimized in vacuo. Like Mocci and Cerioni et al., our optimized structures were further evaluated by a single-point energy calculation. Single-point energy calculations used a polarizable continuum model (PCM) with dichloromethane as our solvent. All optimized structures were shown to be at a minimum via frequency calculations.

The metrical parameters involving the bond distances of the triflate bound to iodine shown in our intermediates are compared against experimental data reported by Altabef et al. ${ }^{[24]}$ Experimental bond lengths for the bound triflate show the S-O bond distance to be $1.544 \AA$, while the $\mathrm{O}=\mathrm{S}$ bond distance is shorter with a distance of $1.421 \AA$. Our optimized geometry shows an S-O bond distance of $1.563 \AA$ and an O=S bond distance of $1.434 \AA$ which is in good agreement with that reported in literature. Similarly, experimental bond lengths in triflate of the S-C bond and C-F bond are reported to be $1.851 \AA$ and $1.329 \AA$, respectively, which remains 
negligible with our observed bond distances of $1.868 \AA$ and $1.328 \AA$. The theoretical method utilized in our study aids in reproducing the geometry for bound triflate supported by experimental results.

Mocci and Cerioni et al. focused on PIDA, not the activated intermediates, and thus is not altered by any ions unlike the activated system we present. Another difference is that their transition state search was converged by a constrained geometry optimization. We made several unsuccessful attempts at finding transition states between our proposed intermediates but decided not to employ the constrained optimization technique. We are investigating more robust approaches for transition state searches, and for this reason, no transition states are reported.

\section{Results and Discussion}

The evaluation of PIDA-activation centered around the intermediates possible in the equilibrium between three structures; Phl(OAc) 2, Phl(OAc)(OTf), and Phl(OTf) $)_{2}$. The possibility of Zefirov's reagent, [(Phl) $\left.{ }_{2} \mathrm{O}\right][2 \mathrm{OTf}]$, was considered, but the stoichiometric addition of TMSOTf and the associated energies evaluated, seemed to indicate that the Zefirov's reagent was not a precursor to the use of a full equivalent of TMSOTf. The theoretical aspects of PIDA-activation are not trivial and showcase the continuum between the ionic and covalent bonding that exists in all chemistry. The trove of T-shaped conformers that exists for PIDA-like intermediates led to the evaluation of a potential energy surface that has many local minima and thus many active species are likely in equilibrium at once. The complexity of the acid activation of PIDA also inevitably leads to zwitterions and elongated bonds that via population analysis contain fractional bond orders.

The initial approach to understanding the activation mechanism was to analyze the separate conformers concerning the planarity of the ligands with the phenyl-group as well as the ligands' planarity with respect to each other. The optimized conformers for PIDA fell into local minima with respect to the co-planarity of the acetate ligands. A $2.6 \mathrm{kcal} / \mathrm{mol}$ difference in energy between conformers was calculated and related to the coplanar rotation of the acetates. The difference between cis and trans variants of the periplanar conformers were negligible in DCM. The lower energy of the coplanar geometry can be explained by the partial donation of the $\sigma$-hole. These results are congruent with those of Mocci and Cerioni et al., and a more detailed analysis can be found in their work. ${ }^{[1]}$ It should be noted that each of the structures calculated for the conformation analysis of PIDA were the result of unconstrained geometry optimizations, thus demonstrating the complexity of falling into a local minimum along the potential energy surface. Likewise, many possibilities of activated conformers also needed to be evaluated. 


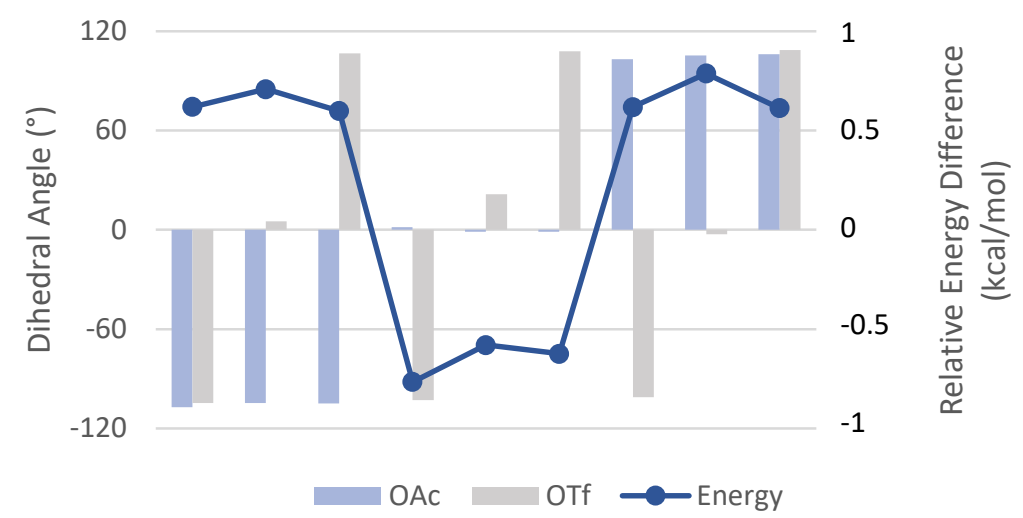

Figure 1. Energy profile related to the degree of rotation for the ligands OAc and OTf showing the negligible effects of OTf conformations as opposed to OAc conformations.

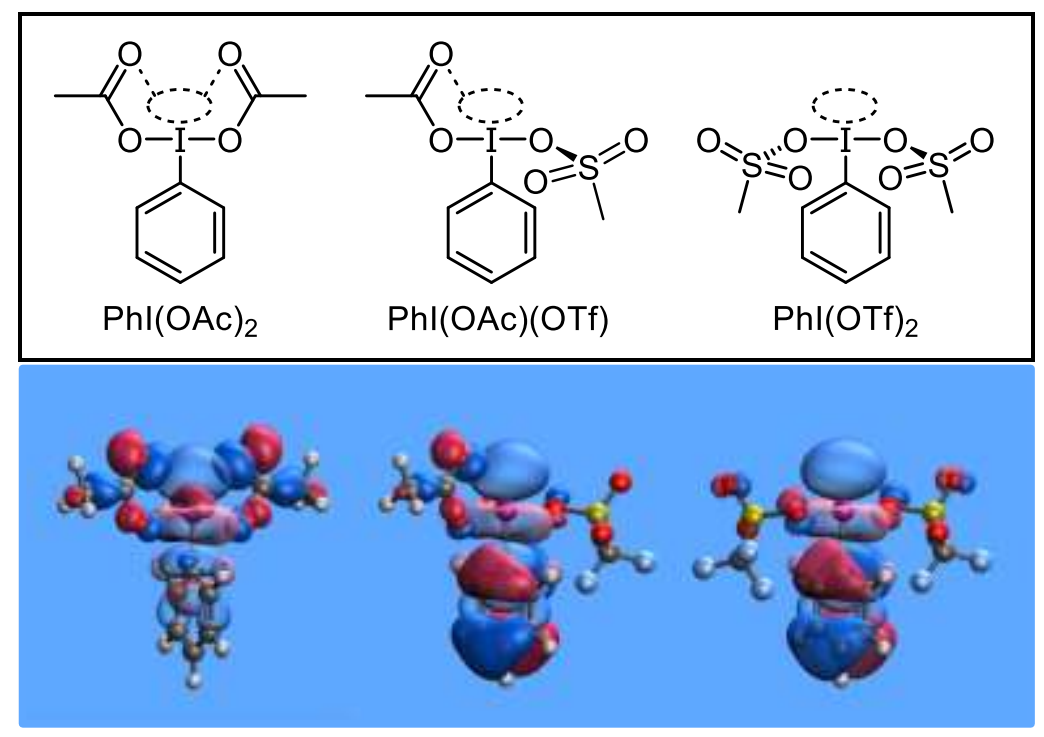

Figure 2. The HOMO-1 overlayed on the LUMO+1 for each structure to show $\sigma$-hole donation.

The correlation between the energies of PhI(OAc)(OTf) and the acetate's coplanar position suggests that, ignoring negligible steric effects, the position of the triflate is mostly irrelevant to the stability of the molecule. This would imply that, while the triflate may orient itself so that the $\mathrm{O}_{\text {lodine }} \mathrm{S}-\mathrm{O}$ bond angle reflects the orientation of the acetate's angle, neither of the triflate's oxygen atoms exhibit significant $\sigma$-hole donation. The energy comparison in Figure 1 and molecular orbital pictures in Figure 2 help demonstrate the difference between the participation of $\sigma$-hole donation of acetate over triflate. Corroborating evidence is also shown in Figure 1 which supports PIDA's preference for both acetates to be coplanar, thus contributing to its optimal orientation to participate in $\sigma$-hole donation. Phl(OAc)(OTf) and Phl(OTf) $)_{2}$ exhibit an opposite energy profile with respect to the triflate conformations. The average difference between the triflate being a coplanar and periplanar conformer is negligible. The conformers are relatively interchangeable and thus there is a higher probability for the sigma-hole to be exposed; a vital component for some HVI reactions. ${ }^{[9]}$

With the results of the conformational analysis we sought to map a reaction coordinate for the activation of PIDA with TMSOTf (Figure 3). Intermediate 2, involves Lewis acid activation of an acetate group on PIDA and its nearly five-coordinate geometry of silicon in which the Si-OAc distance is $3.9 \AA$ and the angles are: TfO-Si-OAc, 
$159^{\circ}$; TfO-Si-Me, $101^{\circ}$; and AcO-Si-Me, $67^{\circ}$. In comparison, a similar intermediate 6 is found after the first acetate-triflate exchange. The Si-OAc distance in intermediate 6 is $3.8 \AA$ and the angles are: TfO-Si-OAc, $177^{\circ}$; TfO-Si-Me, $101^{\circ}$; and AcO-Si-Me, $73^{\circ}$. The slight change closer to a five-coordinate silicon atom is the result of more electron density shifting into the I-OAc, 3c-4e bond that is seen with intermediate 2.

The resultant drop in energy from PIDA (1) to 2 seems to correlate with the findings of Suresh and Sajith on HVI's preferred combinations of how the trans-influence acts upon the $3 \mathrm{c}-4 \mathrm{e}$ bond. ${ }^{[2]}$ Based upon the work by Suresh et al. ${ }^{[2]}$ and Shafir et al. ${ }^{[25]}$ we have evaluated the trans-influence strength of the system to follow the trend of OAC $>$ OAC-TMS > OTf, where OAc has relatively strong trans-influence compared to the weak and very weak trans-influence of the other two ligand options. The classification of trans-influence strength works well to explain the $22 \mathrm{kcal} / \mathrm{mol}$ energy difference between PIDA and intermediates 3 and $\mathbf{4}$. The generation of $\mathrm{Phl}(\mathrm{OTf})_{2}$ (9) from PIDA leads to an overall difference of $22 \mathrm{kcal} / \mathrm{mol}$ as well, and while Suresh and Sajith did not analyze $\mathrm{Phl}(\mathrm{OTf})_{2}(9)$, it can be speculated that the energy difference is due to a reordering of frontier orbitals involved with the $3 c-4 e$ bond.

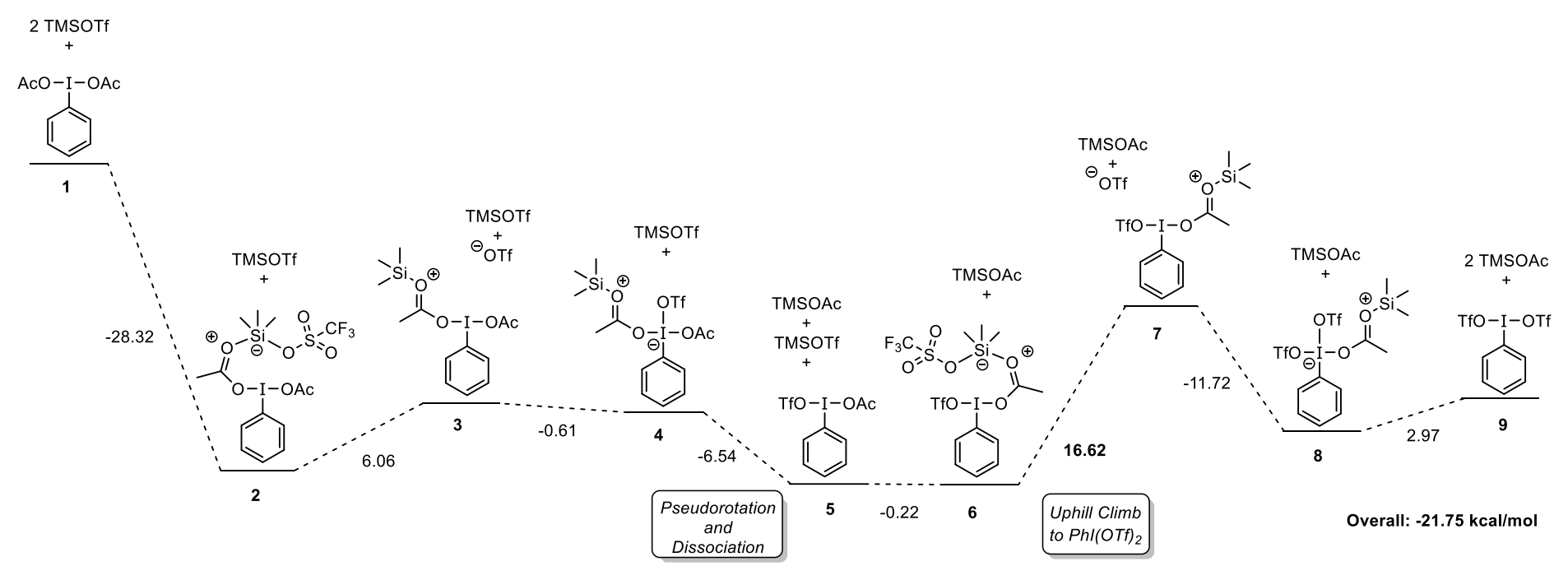

Figure 3. Reaction Coordinate of PIDA Undergoing TMSOTf Activation. Energy barriers are in units kcal/mol.

It seems that along the reaction coordinate shown in Figure 3, the trans-influence is turned off by using triflate ligands. One would expect that the bonding combination of the $3 c-4 e$ bond would result in less stabilization of energy with weak-weak interactions since the 3c-4e non-bonding orbital will be lowered. The HOMO/LUMO gap energy is $120 \mathrm{kcal} / \mathrm{mol}$ for PIDA (1), $107 \mathrm{kcal} / \mathrm{mol}$ for Phl(OAc)(OTf) (5), and $100 \mathrm{kcal} / \mathrm{mol}$ for $\mathrm{Phl}(\mathrm{OTf})_{2}$ (9). If the trans-influence is indeed turned-off with intermediate $\mathbf{9}$, one would expect a restructuring of the frontier orbitals such that the bonding $3 c-4 e$ bond is localized on the $5 p$ orbital of iodine.

The unexpected result of a four-coordinate iodine species (4 and $\mathbf{8}$ ) converged in geometry optimization and shows a difference in stabilization of energy between both intermediates (Figure 3). The minimized geometries of 4 and 8 can be explained by the triflate causing the trans-influence to be turned off upon the TMS-group bonding to the acetate with intermediate 3 . Since acetate itself is not a strong trans-influence ligand, the $3 c-4 e$ bond reorients itself for the strong-weak interaction support by the four-coordinate Ph-I-OTf intermediate, 4. The scenario also holds true for intermediate $\mathbf{8}$ and the difference in energy between intermediate $\mathbf{8}$ and intermediate 4 is less than $2 \mathrm{kcal} / \mathrm{mol}$. 


\section{Conclusions}

The activation of PIDA by TMSOTf was evaluated and found to produce PhI(OAc)(OTf) as the active species although several intermediates are likely in equilibrium. The overall reaction results in approximately a 20 $\mathrm{kcal} / \mathrm{mol}$ lowering of energy upon activation and this energy seems to correlate to the energy difference of the HOMO/LUMO gap when comparing PIDA to the reactive intermediates. The partial filling of the $\sigma$-hole orients the acetate group to be coplanar. The trans-influence is weak when triflate is bound to iodine and thus allows for four-coordinate species that create a new 3c-4e bond along a new coordinate axis. In the active species, $\mathrm{Phl}(\mathrm{OAc})(\mathrm{OTf})$, the triflate conformers are mostly irrelevant to the stability of the molecule as neither of the triflate's oxygen atoms exhibit significant $\sigma$-hole donation.

\section{Experimental Section}

Structure optimizations of the compounds in the reaction coordinate were performed at the DFT level using the popular B3LYP functional in Gaussian '09. ${ }^{[26]}$ The effective core potential for iodine was found from EMSL basis set exchange while the other elements were treated with the $6-311++g(d, p)$ basis set. ${ }^{[27-29]}$ Frequency calculations were performed to confirm each optimized structure was at an energy minimum. The solvent effect has been studied by using a polarizable continuum model (PCM) in single-point energy calculations on the in vacuo optimized geometries. All PCM calculations were carried out at 298.15 K. Graphics of Molecular models were generated using the freely available software: IQmol, Avogadro, and ArgusLab. ${ }^{[30]}$

\section{Acknowledgements}

The research conducted was supported by Adelphi University Faculty Development Grants, Frederick Bettelheim Research Award, and the ACS PRF\# 59555-UNI1. We are grateful to Dr. Andrew L. Sargent and Dr. Lee Bartolotti at East Carolina University for advising the Hyatt Research Group's foray into computational chemistry.

\section{Supplementary Material}

Computational results are available free of charge via the Internet at https://www.arkat-usa.org

\section{References}

1. F. Mocci, G. Uccheddu, A. Frangia, G. Cerioni, J. Org. Chem. 2007, 72, 4163-4168.

https://doi.org/10.1021/jo070111h

2. P. K. Sajith, C. H. Suresh, Inorg. Chem. 2012, 51, 967-977.

https://doi.org/10.1021/ic202047g

3. X.-Y. Chen, C.-S. Zhang, L. Yi, Z.-H. Gao, Z.-X. Wang, S. Ye, CCS Chem. 2019, 1, 343-351.

https://doi.org/10.31635/ccschem.019.20190020

4. A. A. Hussein, Y. Ma, A. Al-Yasari, 2020, DOI 10.26434/CHEMRXIV.12350879.V9. 
5. Z. Qu, H. Zhu, S. Grimme, ChemCatChem 2020, 12, 6186-6190.

https://doi.org/10.1002/cctc.202001392

6. G. Grelier, B. Darses, P. Dauban, Beilstein J. Org. Chem. 2018, 14, 1508-1528.

https://doi.org/10.3762/bjoc.14.128

7. T. P. Pell, S. A. Couchman, S. Ibrahim, D. J. D. Wilson, B. J. Smith, P. J. Barnard, J. L. Dutton, Inorg. Chem. 2012, 51, 13034-13040.

https://doi.org/10.1021/ic302176f

8. F. C. Sousa E Silva, N. T. Van, S. E. Wengryniuk, J. Am. Chem. Soc. 2020, 142, 64-69.

https://doi.org/10.1021/jacs.9b11282

9. O. Kirshenboim, S. Kozuch, J. Phys. Chem. A 2016, https://doi.org/10.1021/acs.jpca.6b07894

10. G. C. Pimentel, J. Chem. Phys. 1951, 19, 446-448. https://doi.org/10.1063/1.1748245

11. A. G. Yurieva, O. K. Poleshchuk, V. D. Filimonov, J. Struct. Chem. 2008, 49, 548-552. https://doi.org/10.1007/s10947-008-0073-9

12. A. S. Ivanov, I. A. Popov, A. I. Boldyrev, V. V. Zhdankin, Angew. Chemie Int. Ed. 2014, 53, 9617-9621. https://doi.org/10.1002/anie.201405142

13. V. V. Zhdankin, Arkivoc 2020, 2020, 1-11. https://doi.org/10.24820/ark.5550190.p011.145

14. M. Ochiai, A. Yoshimura, K. Miyamoto, S. Hayashi, W. Nakanishi, J. Am. Chem. Soc. 2010, 132, $9236-9$. https://doi.org/10.1021/ja104330g

15. V. V. Zhdankin, Hypervalent lodine Chemistry: Preparation, Structure, and Synthetic Applications of Polyvalent lodine Compounds, Wiley: Chichester, 2014.

https://doi.org/10.1002/9781118341155

16. J. Noorollah, H. Im, F. Siddiqi, N. Singh, N. R. Spatola, A. Chaudhry, T. J. Jones, I. F. D. Hyatt, European J. Org. Chem. 2020, 2302-2305 https://doi.org/10.1002/ejoc.202000393

17. C. I. Herrerías, T. Y. Zhang, C. J. Li, Tetrahedron Lett. 2006, 47, 13-17. https://doi.org/10.1016/j.tetlet.2005.10.123

18. J. C. Walters, A. F. Tierno, A. H. Dubin, S. E. Wengryniuk, European J. Org. Chem. 2018, 2018, $1460-1464$. https://doi.org/10.1002/ejoc.201800118

19. B. T. Kelley, J. C. Walters, S. E. Wengryniuk, Org. Lett. 2016, 18, 1896-1899. https://doi.org/10.1021/acs.orglett.6b00672

20. S. D. Houston, L. Sharp-Bucknall, M. Albayer, J. Dutton, _ T., 2020, DOI 10.26434/CHEMRXIV.12645710.V1.

21. Tania, S. D. Houston, L. Sharp-Bucknall, T. B. Poynder, M. Albayer, J. L. Dutton, Chem. - A Eur. J. 2020, chem.202003819.

22. A. Aprile, K. J. Iversen, D. J. D. Wilson, J. L. Dutton, Inorg. Chem. 2015, 54, 4934-4939. https://doi.org/10.1021/acs.inorgchem.5b00537

23. U. Farid, T. Wirth, Angew. Chemie - Int. Ed. 2012, 51, 3462-3465. https://doi.org/10.1002/anie.201107703

24. M. E. Defonsi Lestard, M. E. Tuttolomondo, E. L. Varetti, D. A. Wann, H. E. Robertson, D. W. H. Rankin, A. Ben Altabef, J. Mol. Struct. 2010, 984, 376-382. https://doi.org/10.1016/i.molstruc.2010.10.009

25. S. Izquierdo, S. Essafi, I. del Rosal, P. Vidossich, R. Pleixats, A. Vallribera, G. Ujaque, A. Lledós, A. Shafir, J. 
Am. Chem. Soc. 2016, 138, 12747-12750.

https://doi.org/10.1021/jacs.6b07999

26. D. J. Frisch, M. J.; Trucks, G. W.; Schlegel, H. B.; Scuseria, G. E.; Robb, M. A.; Cheeseman, J. R.; Scalmani, G.; Barone, V.; Petersson, G. A.; Nakatsuji, H.; Li, X.; Caricato, M.; Marenich, A. V.; Bloino, J.; Janesko, B. G.; Gomperts, R.; Mennucci, B.; Hratch, 2009.

27. W. R. Wadt, P. J. Hay, J. Chem. Phys. 1985, 82, 284-298. https://doi.org/10.1063/1.448800

28. K. L. Schuchardt, B. T. Didier, T. Elsethagen, L. Sun, V. Gurumoorthi, J. Chase, J. Li, T. L. Windus, J. Chem. Inf. Model. 2007, 47, 1045-1052.

https://doi.org/10.1021/ci600510j

29. B. P. Pritchard, D. Altarawy, B. Didier, T. D. Gibson, T. L. Windus, J. Chem. Inf. Model. 2019, 59, 4814-4820. https://doi.org/10.1021/acs.jcim.9b00725

30. M. D. Hanwell, D. E. Curtis, D. C. Lonie, T. Vandermeerschd, E. Zurek, G. R. Hutchison, J. Cheminform. 2012, $4,17$.

https://doi.org/10.1186/1758-2946-4-17

This paper is an open access article distributed under the terms of the Creative Commons Attribution (CC BY) license (http://creativecommons.org/licenses/by/4.0/) 